\title{
High-Resolution Observations of Emerging Magnetic Fields and Flux Tubes in Active Region Photosphere
}

\author{
T. Tarbell, S. Ferguson, Z. Frank, R. Shine, A. Title, K. Topka \\ Lockheed Palo Alto Research Laboratory \\ 3251 Hanover Street, Palo Alto, California 94304, USA \\ and G. Scharmer \\ Royal Swedish Academy of Sciences \\ Stockholm Observatory, S-133 00 Saltsjöbaden, Sweden
}

\section{Introduction}

On 29 September 1988, filtergrams of the solar photosphere with excellent resolution ( 0.3 to 0.5 arcsecond) were obtained at the Swedish Solar Observatory on La Palma, Canary Islands. An outstanding 2.5 hour run of digital filtergram observations was obtained, looking at a small area within an active region near disk center. On 6 August 1987, an 80 minute run of similar observations was obtained at the Vacuum Tower Telescope of the National Solar Observatory at Sacramento Peak. Digital and video movies have been made of Dopplergrams, magnetograms, line center, continuum, and white light images. Several examples of magnetic field emergence and formation of flux tubes can be studied in detail in the movies. The relationship between photospheric bright points, "filigree", the line center brightness, and the magnetic field has been established for individual images in analysis to date.

\section{Observations}

The data were collected using an evaluation model of the tunable filter systems developed for the NASA Solar Optical Universal Polarimeter (SOUP) and the Coordinated Instrument Package of the NASA Orbiting Solar Laboratory (OSL). The system (see Figure 1) consists of a high speed steering mirror for image stabilization, reimaging optics, a polarization analyser, a blocking filter wheel, a narrowband (70 $\mathrm{m} \AA$ ) tunable filter, and a $1024 \times 1024 \mathrm{CCD}$ camera that uses a Texas Instruments uniphase detector. During periods of the best seeing, only data from a $512 \times 512$ subarea of the array (about $85 \times 85$ arcseconds) were recorded to increase the frame rate (4-5 seconds per frame). The target region on 29 September was dense plage adjacent to a large sunspot in NOAA active region 5168 at North 18 , West 0 . The 


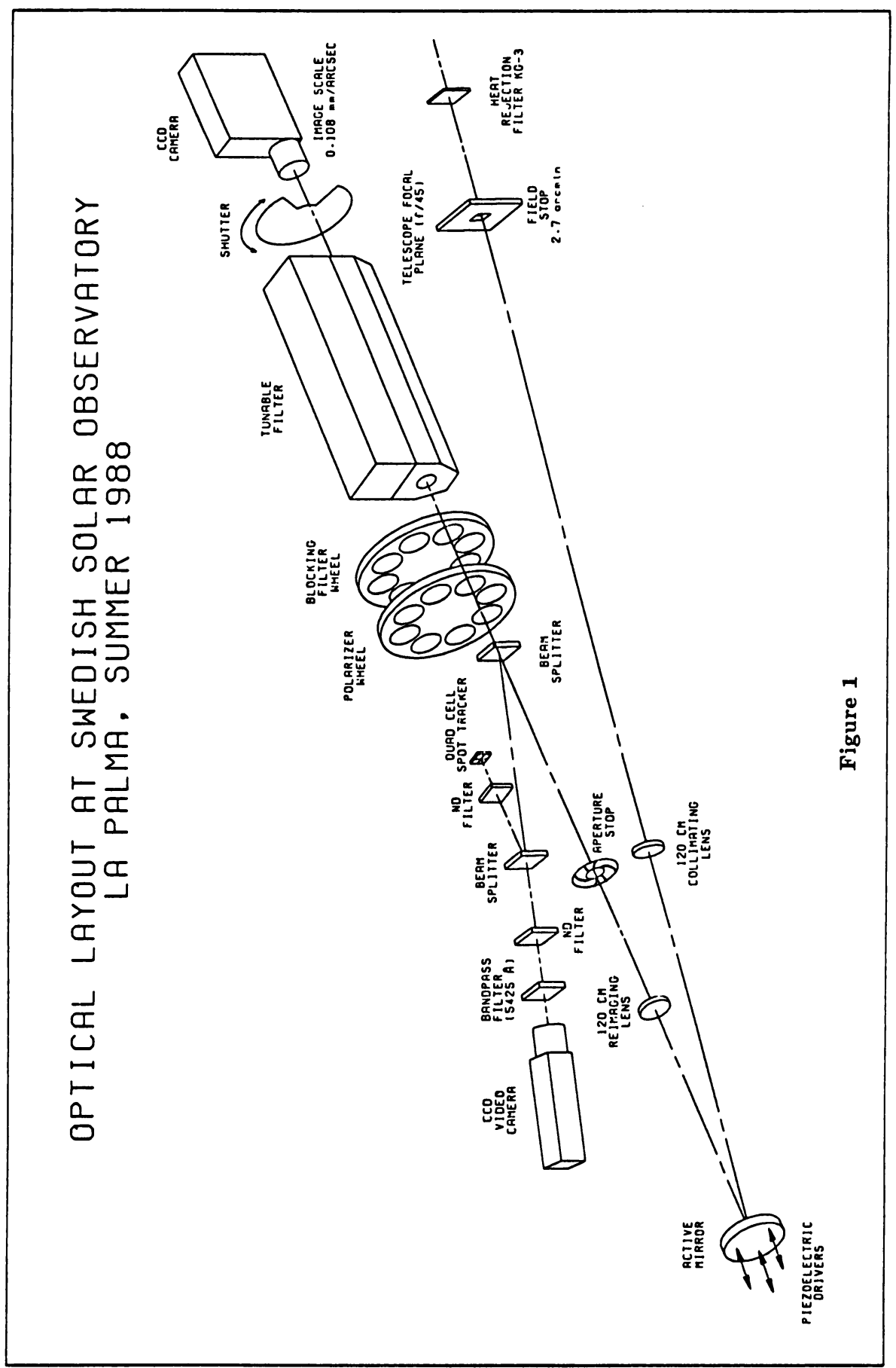


observing sequence had a 50 second cycle and consisted of images in RCP and LCP in the blue wing of Fe I 6302 for longitudinal magnetic measurements, four images spaced through Ni I 6768 for Doppler shift and line center intensity, and continuum images near one or both lines. At Sacramento Peak, we used the $\mathrm{g}=0$ line Fe I 5576 instead of 6768 and included three $\mathrm{H} \alpha$ frames as well, with an overall cycle time of 80 seconds. The field-of-view included most of a small growing active region (NOAA 4835) at South 29, West 11.

Although the raw images make fascinating movies, intensive computer processing is needed to convert them into measurements of physical quantities with $0.3-0.5$ arcsecond resolution (Title et al., 1989a). Removal of spatial image distortions caused by atmospheric turbulence ("destretching") is the most difficult and timeconsuming step (Topka et al., 1986; November, 1986). With a suitable destretching procedure, it is possible to create time series of magnetograms, Doppler velocity, continuum, and line center intensity images for the entire 2.5 hour interval that are essentially simultaneous and aligned to a fraction of the spatial resolution.

\section{Filigree and Magnetic Fields}

Filigree are bright structures seen in the photosphere which are associated with fine scale magnetic fields (Dunn and Zirker, 1973). They range from points to linear structures, typically one or two arcseconds in length, one quarter arcsecond or less in width, and often "crinkled" on an arcsecond scale. Modern analysis of various spectroscopic observations (Stenflo, 1973; see reviews by Muller, 1985, and Stenflo, 1989; see Zirin, 1988, for a dissenting view) suggests that nearly all magnetic fields in the photosphere are in flux tubes too small to be observed directly, with kilogauss field strengths and diameters of order $100 \mathrm{~km}$ (0.14 arcseconds). It has been suggested (Dunn and Zirker, 1973; Mehltretter, 1974) that the filigree bright points are the elementary flux tubes, but some observations of very high quality appear to show the magnetic fields covering a much larger area (Simon and Zirker, 1974).

The 29 September data show many examples of filigree in the continuum and Ni I 6768 line center images. (See the review article by A. Title in this volume for examples of these images.) The magnetic fields outside sunspots have intricate fine structure down to the spatial resolution limit of the images. Some of our conclusions from viewing the movies and from detailed quantitative study of one time step of the 29 September data set are as follows.

(1) Magnetic regions have an "abnormal" granulation as seen in the continuum that is distinctly different from the granulation pattern seen in non-magnetic regions. Granules in the abnormal regions are smaller and the lanes between them are as well. In the photosphere above the abnormal granules, the vertical component of the velocity field is lower on average. Analysis of SOUP movies (Title et al., 1989b) and the Doppler movie from Sacramento Peak (Tarbell et al., 1988) show that this reduction occurs both in the 5 minute oscillations and in the granulation 
velocities.

(2) The bright points observed in the Ni I 6768 line center are related to magnetic field structures. In weakly magnetic regions the bright points are cospatial with equally fine scale magnetic structures. In areas of higher magnetic flux, bright points lie within magnetic structures and are significantly smaller than the magnetic elements.

(3) Bright points in the Ni I 6768 line center are also usually seen as weak local maxima in the continuum intensity. In stronger magnetic areas, the continuum intensity is usually average or below average. The relation between line center and continuum varies considerably, depending on the magnetic flux, indicating varying intrinsic absorption depth of the $\mathrm{Ni} I$ line.

(4) In movies, both the bright points and the magnetic field structures evolve on comparable time scales. However, this does not mean that magnetic field disappears on a time scale of tens of minutes. Rather, magnetic field elements move and change their local configuration.

(5) The magnetic field appears to outline cellular structures of a wide range of sizes. A thresholded magnetogram resembles a fractal set, similar to those in Mandelbrot (1983). We can estimate the fractal dimension of this set from a plot of area versus length scale and find self-similar behaviour with a dimension of about 1.6 over the entire range considered, from 0.16 to 10 arcseconds.

We have made scatterplots of line center intensity versus continuum intensity, for pixels with magnetogram signals in selected bands. These show that the two intensities are correlated at all levels of the magnetogram, but the slopes and intercepts of the regression lines change in a complex way. Below 700 Gauss, the slope is much larger than in non-magnetic areas, indicating a smaller fractional line absorption depth in the magnetic structures. Above 700 Gauss, the slope decreases (line absorption increases), and most of the magnetic pixels are at average or below average intensity in both images. A more detailed study of the four-dimensional relationships between magnetogram signal, Doppler velocity, continuum, and line center intensity will be published elsewhere.

\section{Emerging Magnetic Flux}

Both Sacramento Peak and La Palma movies show many examples of tiny flux tubes emerging through the surface. We learned to recognize the signs of emerging flux in intensity and Doppler movies from 6 August 1987, and so it is now easy to find such events in the 29 September data, which have higher resolution and more uniform seeing. The quantitative discussion below is based on careful analysis of a few events in the 6 August movies (Tarbell et al., 1989). The La Palma events are very similar in the movies but have not yet been studied quantitatively.

The first observable signal for the emergence of new magnetic flux is the formation of dark alignments seen in the continuum and line center. The dark alignments seen in our data are typically 1500 to $4000 \mathrm{~km}$ long and have lifetimes of 5 to 15 
minutes. A strong upflow reaching 0.5 to $0.8 \mathrm{~km} / \mathrm{s}$ may be seen for a few minutes in the dark alignment just after it appears. The upward velocity profile integrated over the duration of the event suggests a thickness of order only $100 \mathrm{~km}$ for the rising flux tube. Bright points form at one or both ends of the dark alignment a few minutes after it appears. Strong downflow reaching $-1.0 \mathrm{~km} / \mathrm{s}$ and new magnetic flux appear in the bright points. The downflow may persist for an hour or more; the mass flux integrated over this time period would empty a tube roughly 3000 $\mathrm{km}$ long at photospheric densities, consistent with the initial footpoint separation. In one event studied most carefully, the magnetic flux appears to increase gradually over a period of at least half an hour, instead of making an abrupt change when the bright point first appears. The observed emergence rate is about $3 \mathrm{x}$ $10^{15} \mathrm{Mx} / \mathrm{s}$. More events must be measured in the more uniform seeing of the 29 September movies to see if this result is confirmed. The footpoints separate at easily measurable horizontal speeds, sometimes as high as $3 \mathrm{~km} / \mathrm{s}$.

Zwaan and Brants (1985) have also seen many of these features in an emerging flux region. Theoretical studies of the emergence process have been published by Moreno-Insertis (1986) for the rise through the convection zone and by Shibata et al. $(1989 \mathrm{a}, \mathrm{b})$ for eruption through the photosphere and expansion into the upper atmosphere. It is clear that a modest increase in resolution and uniformity of seeing would permit meaningful comparisons with MHD models. Continued observations of this type at excellent sites, perhaps with assistance from active optics, or balloon flights of the SOUP instrument could provide such observations in the near future.

ACKNOWLEDGMENTS. This work has been supported by NASA contracts NAS8-32805 (SOUP) and NAS5-26813 (OSL), by Lockheed independent research funds, and by the Royal Swedish Academy of Sciences. The observations were obtained at the Observatorio del Roque de los Muchachos of the Instituto de Astrofisica de Canarias and at the National Solar Observatory in Sunspot, New Mexico.

\section{References}

Dunn, R.B. and Zirker, J.B. (1973) Solar Phys., 33, 281.

Mandelbrot, B. (1983) "The Fractal Geometry of Nature" (W.H. Freeman, San Fransisco), pp. 308-9.

Mehltretter, P. (1974) Solar Phys., 38, 43.

Moreno-Insertis, F. (1986) Astr. Ap., 166, 291.

Muller, R. (1985) Solar Phys., 100, 237.

November, L.J. (1986) Appl. Optics, 25, 392.

Shibata, K., Tajima, T., Matsumoto, R., Horiuchi, T., Hanawa, T., Rosner, R., and Uchida, Y. (1989a) Ap. J., 338, 471.

Shibata, K., Tajima, T., Steinolfson, R.S., and Matsumoto, R. (1989b) Ap. J., in press. 
Simon, G.W., and Zirker, J.B. (1974) Solar Phys., 35, 331.

Stenflo, J.O. (1973) Solar Phys., 32, 41.

Stenflo, J.O. (1989) Astron. Astrophys. Rev., 1, 3.

Tarbell, T., Peri, M., Frank, Z., Shine, R., and Title, A. (1988) in "Seismology of the Sun and Sun-Like Stars," Domingo, V. and Rolfe, E.J. (eds.), European Space Agency SP-286, (ESTEC, Noordwijk, the Netherlands), 315.

Tarbell, T., Topka, K., Ferguson, S., Frank, Z., and Title, A. (1989) in "Proc. of Sac. Peak Summer Workshop on High Spatial Resolution Solar Observations", Luhe, O. von der (ed.), (NSO, Sunspot), in press.

Title, A., Tarbell, T., Topka, K., Cauffman, D., Balke, C., and Scharmer, G. (1989a) in "Physics of Magnetic Flux Ropes", Russell, C.T. (ed.), Am. Geophys. Union, in press.

Title, A.M., Tarbell, T.D., Topka, K.P., Ferguson, S.H., Shine, R.A., and the SOUP Team (1989b) Ap. J., 336, 475.

Topka, K.P., Tarbell, T.D., and Title, A.M. (1986) Ap. J., 306, 304.

Zirin, H. (1988) "Astrophysics of the Sun" (Cambridge Univ. Press, Cambridge), pp. 131-5.

Zwaan, C., and Brants, J.J. (1985) Solar Phys., 95, 3. 